\section{Los caminos de la terminología: una entrevista con Mercè Lorente Casafont}

On the paths of terminology: an interview with Mercè Lorente Casafont

Diego Napoleão Viana AZEVEDO (UFSC) diegonapoleao@gmail.com

Recebido em: 18 de jul. de 2020. Aceito em: 09 de ago. de 2020.

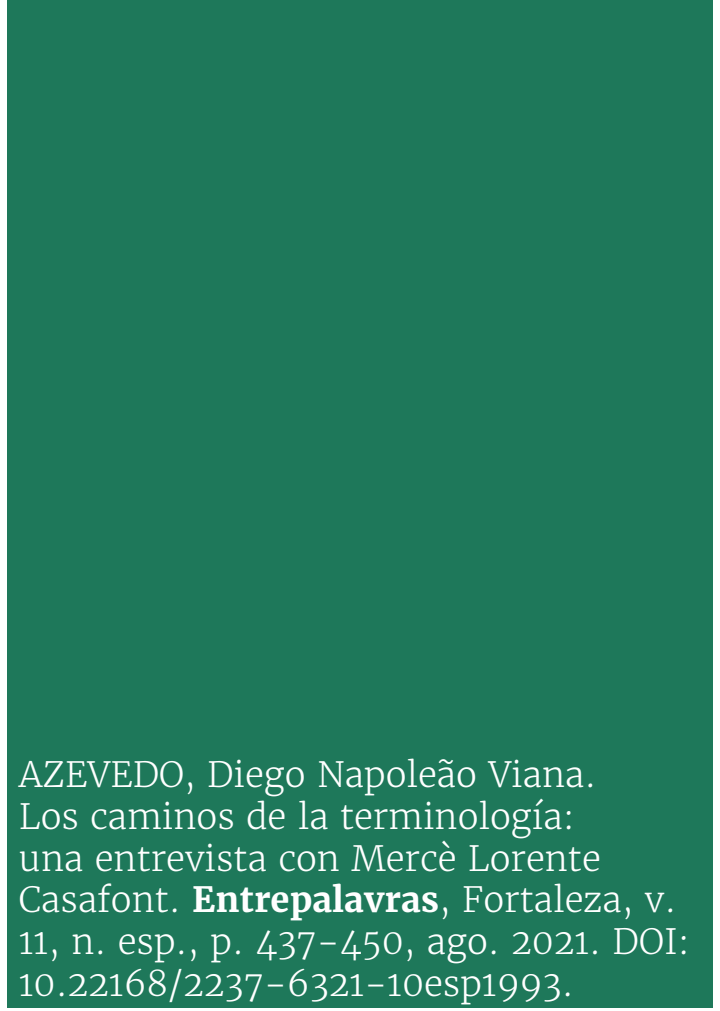

Resumen: En esta entrevista, Mercè Lorente Casafont habla de la evolución de la terminología como disciplina científica desde la perspectiva de la Teoría comunicativa de la terminología (TCT) (CABRÉ, 1999). Lorente revisita su experiencia con la investigación de unidades verbales de carácter terminológico y trata de la actuación del grupo de investigación IULATERM (Léxico y tecnología) para el desarrollo continuo de la TCT, poniendo en relieve las principales investigaciones que expanden y refuerzan ese marco teórico. Además, Lorente presenta un conjunto de herramientas tecnológicas que han elaborado para el procesamiento del léxico y expone la relevancia de los conocimientos terminológicos para la planificación lingüística y para la pervivencia de lenguas minorizadas, como el catalán.

Palabras clave: Teoría comunicativa de la terminología. Aplicaciones terminológicas. IULATERM. 
v. 11 (esp.)

437-450 ago. 2021

Abstract: In this interview, Mercè Lorente Casafont talks about the evolution of terminology as a scientific discipline, especially from the perspective of the Communicative Theory of Terminology (CTT) (CABRÉ, 1999). Lorente recounts her experience with the investigation of verbal units of terminological nature and discusses the contributions of the IULATERM (Lexicon and Technology) research group for the continuous development of the CTT by highlighting the main studies that have expanded and reinforced this theoretical framework. Furthermore, Lorente presents a set of technological tools for lexical processing also developed by this research group and stresses the relevance of terminological knowledge for linguistic planning and for the survival of minority languages, such as Catalan.

Keywords: Communicative Theory of Terminology. Terminological applications. IULATERM.

\section{Introducción}

En esta entrevista, llevada a cabo en Barcelona el 28 de enero de 2019, Mercè Lorente Casafont habla de la evolución de la terminología como disciplina científica y de la experiencia del grupo IULATERM (Léxico y tecnología) para el desarrollo de la Teoría comunicativa de la terminología (TCT).

Mercè Lorente Casafont ${ }^{1}$ es doctora en filología catalana por la Universitat de Barcelona (UB) y profesora titular en la Universitat Pompeu Fabra (UPF) desde 1994, donde imparte docencia, sobre todo, en terminología y lingüística aplicada. Lorente tiene una amplia experiencia profesional en la enseñanza de la lengua catalana, la producción de vocabularios especializados y la investigación en terminología.

Actualmente, Lorente es directora del Institut de Lingüística Aplicada (IULA) de la UPF e investigadora principal del grupo IULATERM (Léxico y tecnología), reconocido internacionalmente por el desarrollo de la Teoría comunicativa de la terminología, bajo el liderazgo de María Teresa Cabré, y por su tradición en la investigación en el ámbito de la terminología, neología, lexicografía especializada y tecnologías del lenguaje. Además, Lorente es miembro del Institut d'Estudis Catalans (IEC) y editora jefe de la revista TERMINÀLIA.

\footnotetext{
${ }^{1}$ El currículo completo de Mercè Lorente Casafont está disponible en: https://www.upf.edu/ web/merce-lorente.
} 


\section{La entrevista}

Diego Napoleão Viana Azevedo (DNVA): Al revisar su currículo, se puede notar que usted tiene una larga y exitosa trayectoria académica y profesional, sobre todo en lo que se refiere a los estudios catalanes y a la terminología. ¿Cómo ha empezado su interés por estos ámbitos?

Mercè Lorente Casafont (MLC): Mis intereses [por estos ámbitos] empezaron cuando estuve estudiando en la Universidad de Barcelona. En aquel momento, no tenía muy claro que quisiera dedicarme a la lingüística. Me gustaba mucho leer, me gustaba muchísimo todo el ámbito de humanidades y empecé pensando que haría latín. A mí me gustaba especialmente la sintaxis y, a través de los estudios, vi que esto podía ser una buena vía. Pero, cuando empecé en la universidad, me di cuenta de que el tipo de estudios que se ofrecían no era lo que me interesaba y me decanté por la filología catalana. En filología catalana, vi que, aunque me gustaba mucho leer, lo mío no era la literatura, sino que la lingüística me interesaba cada vez más. Dentro de la lingüística, lo que más me interesaba era el léxico $\mathrm{y}$, dentro del léxico, fui descubriendo, de la mano de profesores como María Teresa Cabré, qué era la lingüística aplicada. Por tanto, me he movido siempre con mucho placer entre este camino de la teoría lingüística hacia las aplicaciones.

Cuando me vine con María Teresa Cabré a la Universitat Pompeu Fabra y concursé a una plaza de profesora, ya sabía que no iba a hacer docencia sobre lengua catalana estrictamente, sino sobre terminología. En aquel momento, yo aún no conocía demasiado la terminología. Yo había hecho una tesis doctoral sobre léxico, pero sólo léxico general, sobre aspectos sintácticos y semánticos de los verbos en catalán. El léxico me interesaba $\mathrm{y}$, entonces, me empecé a formar con toda la gente del grupo en el ámbito de la terminología. [...] Colaboré en el Servei de Llengua Catalana de la Universitat de Barcelona, coordinando una serie de diccionarios cuya autoría era de catedráticos de las materias: bioquímica de la nutrición, bioquímica clínica [...] y muchos otros temas. Allí aprendí mucha metodología para el trabajo terminológico. También, de la mano de María Teresa Cabré y de mi compañero, Carles Tebé, que éramos los que nos ocupábamos de estos diccionarios, empezamos a observar que había cuestiones metodológicas que no acababan de satisfacernos con la metodología que reinaba en aquel momento. 
v. 11 (esp.)

437-450 ago. 2021

A llegar a la Universitat Pompeu Fabra, el hecho de tener que preparar la docencia en terminología como materia nueva, y además esa experiencia que teníamos antes con el mundo práctico -Teresa Cabré en el TERMCAT y los tres que he mencionado en el Servei de Llengua Catalana-, hizo que, con la ayuda de los nuevos jóvenes (de Judit Freixa, de Rosa Estopà), hiciéramos un seminario. Cada semana nos reuníamos para discutir cuestiones teóricas y metodológicas. De allí salió la idea de la necesidad de proponer una renovación teórica. Teresa Cabré, nuestra maestra y líder, asumió personalmente el reto y todos nosotros la seguimos. [...] Yo ya vine con la tesis hecha, pero los demás estaban con la tesis por hacer y, por tanto, fue un momento muy creativo e interesante de cómo se elabora una teoría y cómo se critica positivamente aquello que no acaba de funcionar. Fue realmente una época muy interesante desde el punto de vista intelectual.

DNVA: Con respecto a la terminología, se sabe que esta es una disciplina todavía muy joven que se ha establecido como tal a principios del siglo pasado, así que tenemos poco menos de cien años de investigación formal en este ámbito. De manera general, ¿cómo está la investigación en terminología hoy en día?

MLC: Seguramente tendremos más novedades [con relación a esto] en el seminario que esta semana nos va a impartir Teresa Cabré2. [...] De las reflexiones y conversaciones que tuvimos en el último congreso de RITERM ${ }^{3}$, nos dimos cuenta cómo se están revitalizando algunos temas y se están iniciando líneas nuevas. Vale la pena pensar y repensar qué líneas están más abandonadas o qué líneas están más paradas y ver cómo nos podemos ayudar para que realmente siga la evolución.

Es verdad que [la terminología] es una materia relativamente nueva. Cien años es poco tiempo para una disciplina, pero hay que tener en cuenta que el cambio de paradigmas más fuerte tuvo lugar en los últimos años. La Teoría general de la terminología de Eugen Wüster ofrecía los fundamentos para una terminología pensada para la normalización industrial y se fue desarrollando a lo largo de treinta años, como mínimo, de su mano hasta que él murió. [...] Pero fue en

\footnotetext{
${ }^{2}$ El seminario se intituló "Avança realment la terminologia? Anàlisi de la situació i reflexió crítica" y fue impartido en la Universitat Pompeu Fabra el 30 de enero de 2019.

3 XVI Simposio de la Red Iberoamericana de Terminología, que ocurrió en la Universidad Autónoma de Manizales (Colombia) en 2018.
} 
las décadas de los setenta y ochenta cuando se empezó a notar que la práctica terminológica necesitaba otros puntos de referencia, no sólo la normalización industrial o la normalización científica. En este sentido, las experiencias en traducción y normalización lingüística en Canadá, la tradición sociolingüística en Francia y en otros países fueron muy enriquecedoras. [...] Las prácticas terminológicas resaltaban que la teoría no se ajustaba exactamente a lo que se necesitaba, pero no se había hecho un replanteamiento teórico. Se continuaba poniendo como referencia la teoría de Wüster. En cambio, fue de la mano de María Teresa Cabré donde realmente el cambio de paradigmas fue más fuerte. Nosotros observamos y abordamos la terminología desde la lingüística, sin negar que haya otras puertas desde las que se puede acceder $\mathrm{y}$, desde la lingüística, la utilizamos como marco general para ver qué elementos del estudio del léxico, de la combinatoria léxica, de la morfología, de la semántica y de la pragmática nos sirven para poder dar cuenta de las características de esas unidades léxicas que adquieren un valor especializado en unos contextos específicos. Por tanto, la terminología contemporánea no tiene cien años. El libro de Teresa Cabré de la Teoría comunicativa (CABRÉ, 1999) aparece a finales de los noventa. Estamos hablando de poco menos de veinte años, y veinte años aquí sí que es muy poco. En veinte años, se han hecho muchas tesis y muchas publicaciones. Ese nuevo pensamiento de María Teresa Cabré ha calado en muchas universidades americanas y europeas $-\mathrm{y}$ españolas también-, pero aún estamos en una materia relativamente joven al lado de la lingüística general, de la sintaxis o de cualquier otra materia que se imparten en las universidades.

\section{DNVA: Acerca más específicamente de sus investigaciones en el campo de la terminología, uno de sus principales intereses son los verbos. ¿Cómo evalúa el estudio de los verbos en la terminología actualmente?}

MLC: Yo creo que aún tenemos margen de actuación, como decía. ¿Por qué? Porque no es el núcleo más importante en los estudios de terminología. Los términos son prototípicamente substantivos. Pero el marco de la teoría comunicativa de la terminología y su aproximación lingüística nos da la posibilidad de poder empezar a hablar de verbos. Una terminología pensada para la normalización [como la Teoría general de la terminología de Wüster], sobre todo, recoge y normaliza unidades terminológicas que son de categoría nominal, que son, como 
v. 11 (esp.)

437-450 ago. 2021

dice Teresa Cabré, las más prototípicas. Pero nuestra aproximación comunicativa muestra que en los textos de manera natural las unidades terminológicas aparecen con otros tipos de unidades léxicas.

Entonces, ¿qué son estas otras piezas? ¿qué son estas unidades léxicas? Lo que intenté hacer yo con mi experiencia en el análisis de verbos era observar si había características sintácticas y semánticas que nos permitieran decir si las unidades léxicas de categoría verbal tienen contenido especializado o no, o si son simplemente relacionales para unas unidades terminológicas de categoría nominal [...]. Hice un intento de clasificación de estas unidades verbales en el año 2007 (LORENTE, 2007). Lo empecé antes en 2004, 2005, y luego la modifiqué en 2007. Llegué a la conclusión de que hay muy pocas unidades verbales que podamos decir que son cercanas a los términos o casi términos. La gran mayoría son unidades de sentido general, unidades léxicas que lo que hacen es estar en relación con unidades terminológicas nominales prototípicas. Eso nos permitía graduar o clasificar los verbos en: verbos que sólo establecen relaciones de carácter discursivo, verbos que establecen relaciones de carácter especializado -porque denotan actividades y procesos de la materia que estamos analizandoy algunos verbos que muy extrañamente tienen una base y sólo esta base es especializada, que no se utiliza en el lenguaje general. Pero la distinción entre el significado general y especializado en los verbos es relativamente pequeña.

¿Por qué nos continúan interesando los verbos? Porque ponen en relación las unidades terminológicas más prototípicas, nos permiten explicar bien la representación del conocimiento en los textos y por otra cosa metodológicamente muy innovadora: en este momento, hay muchas disciplinas que han cambiado su perspectiva didáctica. Pienso ahora en la medicina, por ejemplo, [...] que ha pasado de basarse en estudios muy estáticos y memorísticos -aprender la anatomía, aprender el nombre de los huesos, aprender el nombre de los músculos, de los órganos, de los compuestos químicos- a pasar a trabajar sobre todos los procesos, los cambios y las actividades que se ejercen en el desarrollo de la profesión o en sus investigaciones.

Esto conlleva que, si tú centras la explicación de una ciencia, sobre todo basándose en los procesos, en los cambios y en las relaciones, necesariamente necesitas verbos y necesitas visualizar más estas unidades que, aunque no sean las más prototípicas, son las que van a acompañar precisamente estas unidades en funcionamiento. Por eso, 
ahora una de las tesis que estamos dirigiendo con Rosa Estopà, que está a punto de terminarse, la de Òscar Pozuelo ${ }^{4}$ (2019), es una tesis sobre unidades léxicas eventivas en los textos de especialidad. Esas unidades -verbos y adjetivos - que acompañan unidades terminológicas de categoría nominal crean paradigmas que son interesantes a la hora de hacer la didáctica de una materia, representar una materia, etcétera, y no sólo la lista o la nomenclatura de unidades terminológicas nominales.

DNVA: Además de Directora del Institut de Lingüística Aplicada de la Universitat Pompeu Fabra, usted es la investigadora principal del grupo IULATERM, que es un grupo de investigación internacionalmente reconocido, dedicado al estudio del léxico y sus aplicaciones tecnológicas. ¿Cuáles son las principales líneas de investigación del grupo y cuáles las principales investigaciones actualmente realizadas por él?

MLC: El grupo es grande. Yo pasé a dirigirlo en el momento en que María Teresa Cabré se jubiló como profesora en el 2014. Es un grupo de unos quince o dieciséis doctores más unos quince o dieciséis doctorandos, es decir, estamos siempre sobre la treintena de personas. Estas personas trabajan en líneas distintas, pero complementarias.

Hay una línea histórica de la terminología, que ya hemos comentado, que se relaciona con la docencia y con una parte importante de las investigaciones en proyectos y tesis. La neología es otra gran línea de investigación. ¿Por qué? Porque dentro del grupo está el Observatori de Neologia y, durante muchos años, los proyectos competitivos que recibíamos para investigar también se basaron en la neología general y en la neología especializada en algún caso. Por tanto, estas dos líneas son las que todo el mundo nos reconoce, pero no son las únicas. [...] Además, trabajando en léxico, te encuentras muy a menudo dentro de la línea de lexicografía. La frontera entre lexicografía y terminografía no es clara y, por tanto, también estamos trabajando en lexicografía general. [...] También trabajamos en cuestiones de léxico en general, es decir, morfología, semántica y todos los aspectos que se refieren al análisis del léxico.

Otra línea muy potente en nuestro grupo es la de tecnologías. Tecnologías básicamente para el lenguaje de especialidad y para el léxico de especialidad, la terminología y la neología. Nuestro grupo ha

4 La tesis de Òscar Pozuelo se intitula "L'eventivitat en els textos i en els diccionaris especialitzats en biomedicina" y fue presentada en el 2019. 
v. 11 (esp.)

437-450 ago.

2021

desarrollado diversas herramientas y sistemas de extracción automática de terminología y detección automática de neología. Hemos constituido corpus especializados, desarrollado herramientas de procesamiento para la lengua catalana y para la lengua española. La línea de tecnologías, de recursos lingüísticos, está dirigida por Núria Bel dentro de nuestro equipo. Forman parte todos del grupo IULATERM, pero tienen también sus propias líneas concretas -opinion mining, procesamiento de lenguaje natural, traducción automática- con sus propios proyectos de investigación.

Estas serían más o menos las líneas, aunque no son las únicas. Ahora, por ejemplo, se ha incorporado un profesor que trabaja sobre todo en el seguimiento de la normativa y esa va a ser una línea nueva. Las líneas de investigación se van acoplando a las personas que estamos dentro del grupo, a los intereses del grupo y a los trabajos que en cada momento priorizamos.

\section{DNVA: Creo que ya ha contestado una de mis próximas preguntas acerca de las aplicaciones tecnológicas y herramientas desarrolladas por el grupo. ¿Nos cuenta un poco más sobre eso?}

MLC: En este sentido, el liderazgo de Teresa Cabré ha sido determinante todos estos años, porque, cuando se creó el Institut de Lingüística Aplicada, [...] era el momento en que se estaban creando los grandes corpus nacionales. Teníamos ya el British National Corpus y el Trésor de la langue française y se estaba empezando a trabajar en el Corpus del español en la Real Academia Española y en el Corpus del catalán en el Institut d'Estudis Catalans. Teresa Cabré, al crear el Institut de Lingüística Aplicada, nos propuso a todos los miembros que podríamos hacer un trabajo interesante, no sólo con el desarrollo de herramientas, sino también haciendo unos corpus que fueran claramente especializados, porque esos corpus de referencia nacionales o estatales eran corpus básicamente del lenguaje general. Había alguna cosa de discurso especializado, pero muy poco.

Lo primero que hicimos fue desarrollar herramientas de procesamiento para el catalán y el castellano, y empezamos a construir el corpus de referencia de IULA en derecho, economía, medicina, medioambiente, informática y después se añadió genoma. Ahora se están añadiendo también de otras materias, pero básicamente estos son los corpus por los cuales se nos conoce y son muy usados por 
otros especialistas. Además de las herramientas de procesamiento, se empezaron a diseñar herramientas de extracción -los extractores automáticos de terminología y los detectores automáticos de neología-, la plataforma de trabajo en línea para el Observatori de Neologia y nuestras últimas elaboraciones, que es una herramienta de la que estamos muy orgullosos porque la utilizan muchas universidades españolas y extranjeras: el famoso Terminus $2.0^{5}$, que es una herramienta de gestión de corpus y de terminología.

A diferencia de lo que hay en el mercado, [el Terminus 2.0] es una herramienta que elabora toda la cadena de trabajo terminológico, desde la constitución de corpus y la explotación de estos corpus hasta la previsión de las fichas y la exportación del diccionario del banco de datos terminológico que queramos publicar en internet. Por tanto, es una herramienta muy interesante, pero no es la única. También hubo una herramienta que está a disposición del público, que es ESTEN ${ }^{6}$, para el seguimiento, sobre todo, de variantes neológicas normalizadas y espontáneas, otro tipo de estudios sobre léxico. Se han ido desarrollando herramientas y, sobre todo, mejorando las prestaciones de las herramientas de extracción, como YATE7, y de otros materiales, como puede ser WikiYATE ${ }^{8}$, que es una versión avanzada del extractor automático que tenemos en nuestro centro.

DNVA: Como había dicho, Teresa Cabré ha planteado su teoría a finales de los años noventa que sirve hoy en día como un marco teórico alternativo a la teoría clásica de Wüster en la investigación terminológica. Así que la TCT es una teoría integradora y ha sido desarrollada sobre todo por los miembros del IULATERM. ¿Así que cuáles fueron las investigaciones claves para el desarrollo de esta teoría en el grupo?

MLC: Básicamente las primeras tesis que estaban haciéndose en paralelo al desarrollo de la teoría ya abrieron de alguna manera un poco el panorama. [...] La tesis de Rosa Estopà (1999) -conjuntamente con la de Jorge Vivaldi (2001) después - era una tesis encaminada sobre todo a reconocer e identificar automáticamente aquello que

\footnotetext{
${ }^{5}$ Disponible en: http://terminus.iula.upf.edu/cgi-bin/terminus2.0/terminus.pl.

${ }^{6}$ Disponible en: http://esten.iula.upf.edu/.

${ }^{7}$ Disponible en: http://eines.iula.upf.edu/cgi-bin/Yate-on-the-Web/yotwMain.pl.

${ }^{8}$ Disponible en: http://eines.iula.upf.edu/WikiYATE/wikiYate.html.
} 
v. 11 (esp.)

437-450 ago. 2021

es un término de aquello que no lo es y, por tanto, a partir de ahí poder desarrollar un extractor automático de terminología. La tesis doctoral de Judit Freixa (2002) -otra de las profesoras del centroes muy importante porque de alguna manera abordó en su máxima posibilidad las causas de la variación, que había sido uno de los principios negados en la terminología clásica. [En la Teoría general de la terminología] había que eliminar la variación mientras que en nuestro planteamiento [la Teoría comunicativa de la terminología] decimos que la variación es inevitable porque es lenguaje $\mathrm{y}$, por tanto, lo que hay que hacer es explicarla. Y esto es lo que hizo Judit Freixa con su tesis.

A partir de aquí, las tesis más interesantes o que aportaron y que reforzaron las ideas de Teresa [Cabré], sobre todo, están relacionadas con la variación textual - la de Ona Domènech (2006)- o con las relaciones conceptuales entre términos, que abordó Judit Feliu (2004). [...] Son ejemplos de tesis, pero no se acaba aquí, porque de temas hay muchísimos. Tal vez esas primeras tesis ahora tendrían que complementarse seguramente con tesis que refuercen aspectos pragmáticos o semánticos de la terminología. Además de la tesis de Judit Freixa, hay dos tesis doctorales dentro del grupo que han trabajado los aspectos de variación. [...] Irina Kostina (2010) y Andreína Aldestein (2007) han trabajado sobre todo la variación semántica -la polisemia - y, en el caso de Sabela Fernández Silva (2011), su tesis era sobre la variación denominativa con consecuencias conceptuales. Por tanto, la línea esa iniciada por Judit Freixa explicando las causas de la variación se ha ido desarrollando con tesis más específicas sobre tipos de variación.

Hay otra tesis interesante que creo que es un tema no cerrado del todo donde se abordó sobre todo la variación oral y escrita en lenguajes de especialidad: la de Natalia Seghezzi (2011). Y esta tesis realmente es innovadora en este sentido, porque los textos orales no han sido habitualmente objeto del estudio en terminología y hay que ver si los expertos realmente plantean variación cuando hablan o cuando escriben. Eso es lo que ella intentó hacer con su tesis, pero evidentemente es un experimento costoso y hay que reproducirlo o ampliarlo a otras parejas de lenguaje oral y lenguaje escrito en otros ámbitos de los que ella trabajó. 
DNVA: Y, como todavía la TCT está en desarrollo, ¿qué aspectos cree que todavía necesitan desarrollarse en este marco teórico?

MLC: Antes lo decía. Yo creo que aún tenemos mucho margen para trabajar aspectos de la semántica y de la semántica léxica, porque se han abordado cuestiones del valor terminológico relacionado con la polisemia, la variación conceptual, las relaciones, pero aún tenemos espacio para hablar de algunas cuestiones en semántica que no se han abordado, como cuestiones ideológicas, pragmáticas y culturales en contraste con lenguas, y esto aún no lo hemos podido trabajar en nuestras tesis doctorales.

Hay ámbitos también de la sintaxis que tal vez nos puedan dar pistas para explicar sobre todo los sintagmas, las creaciones sintagmáticas, que son muy habituales en las formaciones léxicas en terminología. Hay tesis que han trabajado sobre los verbos y sobre las estructuras de sintagmas, pero no se ha trabajado ni en todas las lenguas ni con todos los aspectos trabajados, porque las tesis tienen como característica que se profundizan mucho en un tema, pero siempre quedan aspectos que el tema no acaba de tocar $\mathrm{y}$, por tanto, siempre hay espacio para retomar.

Como decíamos, esto nos obliga de alguna manera a ir pensando que es de lo que no hemos hablado, aunque siempre estamos pendientes también de lo que quieren hacer los doctorandos y este es un debate en el que tal vez tengamos que tener una voz más contundente. Es decir, "Bueno, que estas tesis hay que hacerlas ya", y alguien las hará (risas).

DNVA: Como sabemos la terminología es una disciplina interdisciplinar y que mantiene una estrecha relación con otras áreas. Por ejemplo, la terminología puede ser una gran aliada de la planificación lingüística y del mantenimiento de las lenguas minorizadas, como el catalán. ¿Nos puede contar cómo ha contribuido la terminología en este sentido en el contexto catalán?

MLC: [La terminología es] más que interdisciplinar. Teresa Cabré nos ilustró en este sentido, distinguiendo lo que eran disciplinas o interdisciplinas de lo que es un objeto transdisciplinar, es decir, un objeto que podemos abordar desde diversas perspectivas. Nosotros la abordamos desde la lingüística, pero también desde la lingüística aplicada. Por tanto, tenemos que describir y explicar cómo es la 
v. 11 (esp.)

437-450 ago. 2021

terminología. Esta es la parte que se acerca más a la lingüística teórica, pero después tenemos que hacer cosas con la terminología, y esto es lo que nos acercaría a la lingüística aplicada.

Dentro de la lingüística aplicada, es evidente lo que dice. La planificación lingüística y la normalización de las lenguas, sobre todo minorizadas, necesitan de la terminología como un elemento fundamental para que la lengua sirva para todo, es decir, no puede ser una 'lengua de futuro' una lengua que no sirva para todos los escenarios de desarrollo y de vida. Para que la lengua sea viva, debemos tener la disponibilidad léxica en todos los ámbitos del saber. Por tanto, los ámbitos profesionales, los ámbitos universitarios y la investigación necesitan tener su vocabulario [...] si se quiere trabajar en esta lengua.

Esto ha hecho que las lenguas minorizadas prioricen, dentro de sus planes de normalización, la creación de centros de normalización terminológica que van trabajando para poner, a disposición de los hablantes, vocabularios específicos o simplemente materiales especializados en la propia lengua. El problema que siempre tienen las lenguas minorizadas es que el ámbito de especialización profesional y académico suele ser el ámbito donde más fácilmente entran préstamos. Entonces, tenemos que ver si esos préstamos se adaptan o si tenemos alternativa [léxica] en lengua propia. La clave está en que los hablantes puedan continuar hablando y escribiendo, si quieren, en su lengua para cualquier ámbito de especialidad. En el caso de la lengua catalana, esto fue clave para su normalización. Precisamente eso lo tuvieron muy claro los políticos cuando decidieron crear el Centro oficial de la terminología catalana, el TERMCAT. También lo vieron en Canadá con la normalización del francés en Québec. Si una lengua quiere normalizarse en todos sus usos, una parte crucial es la terminología. Pero curiosamente esto [la creación de un centro para la normalización] ha podido hacer pensar, desde fuera, que el catalán no disponía de recursos terminológicos y ¡no!: sí que disponía de recursos, porque los hablantes y los profesores universitarios ya habían empezado esta revitalización en los años setenta, antes de la creación del TERMCAT incluso. La clave fue el cambio de lengua en las aulas.

Los hablantes no han dejado nunca de hablar catalán, pero es evidente que los especialistas recibían su docencia en la época franquista -en la dictadura- exclusivamente en castellano. Solo leían textos en castellano o en otras lenguas extranjeras; nunca en catalán. El uso que tenían del catalán era sobre todo un uso familiar o coloquial, pero 
no un uso cercano a lo que sería la alta especialización. Por lo tanto, cuando las universidades empezaron a incorporar docencia en catalán con la llegada de la democracia, se empezaron también a desarrollar vocabularios específicos de las diversas materias. Luego, poco a poco, las universidades en colaboración con el TERMCAT siguieron desarrollando materiales de manera regular. La pervivencia del catalán está en las manos de sus hablantes, pero el hecho de que la lingüística o la política ayude a que los hablantes dispongan de materiales siempre de alguna manera facilita las cosas.

\section{Consideraciones finales}

En esta entrevista, Mercè Lorente Casfont ha trazado con maestría y precisión un panorama de la evolución de los estudios terminológicos desde el punto de vista de la Teoría comunicativa de la terminología (TCT), señalando desde las razones que la motivaron hasta los esfuerzos colectivos del IULATERM (Léxico y tecnología) para el desarrollo de ese marco teórico.

Al presentar los principales estudios y aplicaciones en el ámbito de la TCT realizados por el grupo IULATERM, Lorente aún ofrece un rol de posibilidades que pueden contribuir para la fundamentación teórica y metodológica de otras investigaciones terminológicas que abordan la terminología desde una perspectiva comunicativa, sin olvidar de hacer mención a la capacidad de expansión y refinamiento de la propia teoría.

De esta manera, Lorente destaca la relevancia de la TCT como paradigma alternativo a la teoría clásica de Wüster en los estudios terminológicos y apunta algunos motivos por los cuales la TCT ha influenciado una gran cantidad de investigaciones y aplicaciones terminológicas a nivel internacional.

\section{Referencias}

ALDESTEIN, A. Unidad léxica y significado especializado: modelo de representación a partir del nombre relacional madre. 2007. Tesis - Institut Universitari de Lingüística Aplicada, Universitat Pompeu Fabra, Barcelona, 2007. Disponible en: <https://www.tesisenred.net/handle/10803/7505>. Acceso en: 23 abr. 2020.

CABRÉ, M. T. La terminología. Representación y comunicación: una teoría de base comunicativa y otros artículos. Barcelona: Institut Universitari de Lingüística Aplicada de la Universitat Pompeu Fabra, 1999.

DOMÈNECH, O. Textos especialitzats i variació vertical: la diversitat 
v. 11 (esp.)

437-450 ago. 2021

terminològica com a factor discriminant del nivell d'especialització d'un text. 2006. Tesis - Institut Universitari de Lingüística Aplicada, Universitat Pompeu Fabra, Barcelona, 2006. Disponible en: <https://www.tesisenred.net/ handle/10803/7503 >. Acceso en: 23 abr. 2020.

ESTOPÀ, R. Extracció de terminologia: elements per a la construcció d'un SEACUSE (Sistema d'Extracció Automàtica de Candidats a Unitats de Significació Especialitzada). 1999. Tesis - Institut Universitari de Lingüística Aplicada, Universitat Pompeu Fabra, Barcelona, 1999. Disponible en: < https:// www.tesisenred.net/handle/10803/7489\#page=1>. Acceso en: 22 abr. 2020.

FELIU, J. Relacions conceptuals i terminologia: anàlisi i proposta de detecció semiautomática. 2004. Tesis - Institut Universitari de Lingüística Aplicada, Universitat Pompeu Fabra, Barcelona, 2004. Disponible en: <https://www. tesisenred.net/handle/10803/7494\#page=1>. Acceso en: 23 abr. 2020.

FERNÁNDEZ SILVA, S. Variación terminológica y cognición: factores cognitivos en la denominación del concepto especializado. 2011. Tesis - Institut Universitari de Lingüística Aplicada, Universitat Pompeu Fabra, Barcelona, 2011. Disponible en: <https://www.tesisenred.net/handle/10803/22638>. Acceso en: 23 abr. 2020.

FREIXA, J. La variació terminològica: anàlisi de la variació denominativa en textos de diferent grau d'especialització de l'àrea de medi ambient. 2002. Tesis - Institut Universitari de Lingüística Aplicada, Universitat Pompeu Fabra, Barcelona, 2002. Disponible en: <https://www.tesisenred.net/ handle/10803/1677\#page=1>. Acceso en 22 abr. 2020.

KOSTINA, I. La variación conceptual de los términos en el discurso especializado. 2010. Tesis - Institut Universitari de Lingüística Aplicada, Universitat Pompeu Fabra, Barcelona, 2010. Disponible en: <https://www. tesisenred.net/handle/10803/7513>. Acceso en: 23 abr. 2020.

LORENTE, M. Les unitats lèxiques verbals dels texts especialitzats: redefinició d'una proposta de classificació. In: LORENTE, M.; ESTOPÀ, R.; FREIXA, J.; MARTÍ, J.; TEBÉ, C. (ed.). Estudis de lingüística i de lingüística aplicada en honor de M. Teresa Cabré Castellví. Vol. 2. Barcelona: Institut Universitari de Lingüística Aplicada. Universitat Pompeu Fabra; 2007, p. 365-380.

POZUELO, O. L'eventivitat en els textos i en els diccionaris especialitzats en biomedicina. Tesis - Institut Universitari de Lingüística Aplicada, Universitat Pompeu Fabra, Barcelona, 2019. Disponible en: <https://www. tdx.cat/handle/10803/667927>. Acceso en: 22 abr. 2020.

SEGHEZZI, N. Variación terminológica y canal de comunicación: estudio contrastivo de textos especializados escritos y orales sobre lingüística. 2011. Tesis - Institut Universitari de Lingüística Aplicada, Universitat Pompeu Fabra, Barcelona, 2011. Disponible en: <https://www.tesisenred.net/ handle/10803/52066>. Acceso en: 23 abr. 2020.

VIVALDI, J. Extracción de candidatos a términos mediante combinación de estrategias heterogéneas. 2001. Tesis - Universitat Politècnica de Catalunya, Barcelona, 2001. Disponible en: $<$ https://dialnet.unirioja.es/ servlet/tesis? codigo=11172>. Acceso en: 22 abr. 2020. 